\title{
On the Relaxed States in the Mixture of Degenerate and Non-Degenerate Hot Plasmas of Astrophysical Objects
}

\author{
N.L. Shatashvili ${ }^{1,2}$ - S.M. Mahajan ${ }^{3}$ • \\ V.I. Berezhiani ${ }^{1,4}$
}

\begin{abstract}
It is shown that a small contamination of a relativistically hot electron component can induce a new scale (for structure formation) to a system consisting of an ion-degenerate electron plasma. Mathematically expression of this additional scale length is the increase in the index of quasi-equilibrium BeltramiBernoulli states that have been invoked to model several astrophysical systems of interest. The two species of electrons, due to different origin of their relativistic effective masses, behave as two distinct components (each with its own conserved helicity) and add to the richness of the accessible quasi equilibrium states. Determined by the concrete parameters of the system, the new macro-scale lengths (much larger than the short intrinsic scale lengths (skin depths) and generally much shorter than the system size) open new pathways for energy transformations.
\end{abstract}

Keywords Stars: evolution; stars: atmospheres; stars: white dwarfs; stars: jets; plasmas

\section{Introduction}

In many compact astrophysical objects, the plasma density is so high that the mean inter-particle distance

\section{N.L. Shatashvili}

${ }^{1}$ Andronikashvili Institute of Physics, TSU, Tbilisi 0177, Georgia

${ }^{2}$ Department of Physics, Faculty of Exact and Natural Sciences, Ivane Javakhishvili Tbilisi State University (TSU), Tbilisi 0179, Georgia

S.M. Mahajan

${ }^{3}$ Institute for Fusion Studies, The University of Texas at Austin, Austin,Tx 78712

V.I. Berezhiani

${ }^{1}$ Andronikashvili Institute of Physics, TSU, Tbilisi 0177, Georgia

${ }^{4}$ School of Physics, Free University of Tbilisi, Georgia becomes smaller than the De Broglie thermal wavelength. The Fermi Energy for such a highly degenerate gas (obeying Fermi-Dirac statistics) can become relativistic, especially for the lighter electrons. Consequently, the degeneracy pressure may easily dominate the thermal one [see e.g. (Shapiro \& Teukolsky 1973), (Michel 1982; Berezhiani et al 2015a),

(Berezhiani et al 2015b; Shatashvili et al 2016) and references therein]. Relativistic outflows/jets, often, come out of these compact objects. The interaction of the Relativistic ejecta with their degenerate shells could create turbulence and shock waves at short scales. On the large scale, however, one could still expect the interacting system to be describable in terms of quasi equilibrium states. An interesting representative of such systems is a White Dwarf (WD) - the most common endpoint of stellar evolution Koester \& Chanmugam 1990; Liebert et al 2003),

(Schmidt et al 2003; Kawka et al 2007),

(Külebi et al 2009; Kepler et al 2013),

(Kawka \& Vennes 2014; Hollands et al 2015).

Since many stars are born in binary systems with sufficiently small initial separations, they go through one or more phases of mass-exchange (see e.g. Winget \& Kepler 2008; Tremblay et al 2015), (Mukai 2017) and references therein). The solar neighborhood is populated by numerous accreting white dwarfs (AWDs) that are surrounded by an accretion gas of companion star or disk (Begelman et al 1984; Mukai 2017). The composite system is a highly interesting and unusual state of matter; a highly degenerate WD plasma co-existing with a classical hot accreting astrophysical flow.

Recently it was shown that accreting WD atmospheres may support degenerate plasma relaxed states (Berezhiani et al 2015a) that have associated fast super-Alfvénic up-flows with dramatically reduced densities (Barnaveli \& Shatashvili 2017). It is expected 
that this combination - a bulk degenerate plasma contaminated by small fraction of a non-degenerate highly relativistic plasma - will also pertain during the relativistic jet formation from accretion-induced collapsing White Dwarfs to Black Holes (Begelman et al 1984; Krivdyk 1999; Kryvdik \& Agapitov 2007).

Following the conceptual framework developed in the study of multi-component Relaxed States (including relativistic ones) [see e.g. (Mahaian \& Yoshida 1998; Yoshida \& Mahajan 1999; Mahajan et al 2001), (Oliveira \& Taiima 1995; Steinhauer \& Ishida 1997), (Morrison 1998; Yoshida et al 2001), (Iabal et al 2008; Pino et al 2010; Mahajan \& Lingam 2015),

(Dennis et al 2014; Shatashvili et al 2016) and references therein], we will study, in this paper, the quasi equilibrium states that are accessible to the system composed of two electron species (a highly degenerate main component (to be called $d$ ) mixed with a smaller classical relativistic flow (to be called $h$ )) immersed in a neutralizing ion background; the latter could be either static or dynamic. One of the principal aims of such a search is to extract any new scales of structure formation induced by the addition of new physics to the system. One will, thus, look for the $h$ component induced, intermediate macroscopic length scale $L_{\text {macro }}\left[L_{\text {meso }}\right]$ lying between the system size and the relatively small intrinsic scales (measured by the skin depths); a knowledge of these scales may help us better understand the evolution of accreting stars.

\section{Model Equations}

We will be studying a quasi neutral plasma of a mobile classical ion component $(i)$, and two relativistic electron species - the bulk $d$ electron gas with a density $N_{0 d}$ and a small contamination of $h$ electrons with density $N_{0 h}$. The quasi neutrality demands

$N_{0 d}+N_{0 h}=N_{0 i} \Longrightarrow \frac{N_{0 i}}{N_{0 d}}=1+\alpha, \quad \alpha \equiv \frac{N_{0 h}}{N_{0 d}}$,

where $\alpha \ll 1$ labels the ratio of hot electron fraction. to the degenerate electrons.

The electron dynamics for both components will be described by the appropriate relativistic fluid equations [(Pino et al 2010), (Berezhiani et al 2015a),

(Berezhiani et al 2015b)]: the continuity

$\frac{\partial N_{d(h)}}{\partial t}+\nabla \cdot\left(N_{d(h)} \mathbf{V}_{d(h)}\right)=0$ and the equations of motion:

$$
\begin{aligned}
& \frac{\partial}{\partial t}\left(G_{d(h)} \mathbf{p}_{d(h)}\right)+m_{e} c^{2} \nabla\left(G_{d(h)} \gamma_{d(h)}\right) \\
& =-e \mathbf{E}+\mathbf{V}_{\mathbf{d}(\mathbf{h})} \times \Omega_{d(h)}
\end{aligned}
$$

where $\mathbf{p}_{d(h)}=\gamma_{d(h)} m_{e} \mathbf{V}_{d(h)}$ is the hydrodynamic momentum, $n_{d(h)}=N_{d(h)} / \gamma_{d(h)}$ is the rest-frame particle density $\left(N_{d(h)}\right.$ denotes the laboratory frame density) of the degenerate (hot) electron fluid element, $\mathbf{V}_{d(h)}$ is the fluid velocity, and $\gamma_{d(h)}=\left(1-V_{d(h)}^{2} / c^{2}\right)^{-1 / 2}$.

Notice that the factors $G_{d}$ and $G_{h}$, constituting what could be seen as the effective mass, are quite different for the two electron species. In particular, $G_{d}=w_{d} / n_{d} m_{e} c^{2}$, where $w_{d}$ is an enthalpy per unit volume, originates from degeneracy rather than relativistic kinematics. The general expression for enthalpy $w_{d}$ for arbitrary density and temperature (for a plasma described by local DiracJuttner equilibrium distribution function) can be found in (Cercignani \& Kremer 2002). For a fully (strongly) degenerate electron plasma, however, this very tedious expression smoothly transfers to the one with just density dependence: $w_{d} \equiv w_{d}(n)$ (Berezhiani et al 2015a). In fact $w_{d} / n_{d} m_{e} c^{2}=\left(1+\left(R_{d}\right)^{2}\right)^{1 / 2}$, where $R_{d}$ $\left[=\left(n_{d} / n_{c}\right)^{1 / 3}\right.$ with $n_{c}=5.9 \times 10^{29} \mathrm{~cm}^{-3}$ being the critical number-density]. The effective mass factor, then, is simply determined by the plasma rest frame density, $G_{d}=\left[1+\left(n_{d} / n_{c}\right)^{2 / 3}\right]^{1 / 2}$ for arbitrary $n_{d} / n_{c}$. For relativistically hot plasma an expression for effective mass factor $G_{h}$ can be found in (Berezhiani \& Mahajan 1994,1995; Ryu et al 2006).

On taking the curl of these equations, one can cast them into an ideal vortex dynamics ((Mahajan 2003, 2016) and references therein)

$$
\frac{\partial}{\partial t} \boldsymbol{\Omega}_{d(h)}=\nabla \times\left(\mathbf{V}_{\mathbf{d}(\mathbf{h})} \times \boldsymbol{\Omega}_{\mathbf{d}(\mathbf{h})}\right)
$$

in terms of the generalized (canonical) vorticities $\boldsymbol{\Omega}_{\mathbf{d}(\mathbf{h})}=-(e / c) \mathbf{B}+\nabla \times\left(G_{d(h)} \mathbf{p}_{d(h)}\right)$. Note that generalized vorticity would acquire an additional term for isentropic systems [see (Mahajan 2016)]; the present derivation pertains only to the homentropic plasmas. For special astrophysical conditions, canonical vorticity would also have a quantum-mechanical part [see (Mahajan \& Asenjo 2011; Asenjo \& Mahajan 2015) for spinning plasmas], even a general relativistic component [see e.g. (Bhattacharjee et al 2015) for Black Hole accretion disks] in addition to the electromagnetic, kinetic and thermal contributions. For most applications these corrections are negligibly small. Numerical estimates of spin-magnetic interactions of two-fluid plasmas of white dwarfs and neutron stars was presented 
in (Gomez \& Kandus 2018), and was shown to be not so consequential.

Ion fluid dynamics is described by the corresponding Continuity Equation and the following Equation of Motion:

$$
\begin{aligned}
m_{i}\left[\frac{\partial \mathbf{V}_{i}}{\partial t}+\left(\mathbf{V}_{i} \cdot \nabla\right) \mathbf{V}_{i}\right] & =-\frac{1}{N_{i}} \nabla p_{i}+ \\
& +e \mathbf{E}+\frac{e}{c} \mathbf{V}_{i} \times \mathbf{B}
\end{aligned}
$$

The low frequency dynamics is, now, closed with Ampere's law

$$
\nabla \times \mathbf{B}=\frac{4 \pi e}{c}\left[(1+\alpha) N_{i} \mathbf{V}_{i}-N_{d} \mathbf{V}_{d}-\alpha N_{h} \mathbf{V}_{h}\right]
$$

another relation between $\mathbf{V}_{i}, \mathbf{V}_{d(h)}$ and B. Notice that the small hot electron population, represented by $\alpha$ and $\mathbf{V}_{h}$, will become the source of a new scale-length; Finding and exploring this scale length (which adds the diversity to the scale-hierarchy of multi-component plasmas met in astrophysical conditions) is the principal objective of this paper.

We will concentrate on a special class of equilibria known as the Beltrami-Bernoulli (BB) states (Berezhiani et al 2015a). We expect to find the new channel for energy transformations in such a mixture of relativistic plasmas often emerged while the evolution of certain astrophysical objects, specifically while the evolution of accreting stars; star collapsing and etc..

\section{Equilibrium States in 2-temperature relativistic degenerate electron-ion Plasma}

In this paper, the density is normalized to $N_{0 d}$ (the corresponding rest-frame density is $n_{0 d}$ ); the magnetic field is normalized to some ambient measure $\left|\mathbf{B}_{0}\right|$; hot electron gas temperature is normalized to $m_{e} c^{2}$; all velocities are measured in terms of the corresponding Alfvén speed $V_{A}=V_{A d}=B_{0} / \sqrt{4 \pi n_{0 d} m_{e} G_{0 d}}$; all lengths [times] are normalized to the "effective" degenerate electron skin skin depth $\lambda_{\text {eff }}\left[\lambda_{\text {eff }} / V_{A}\right]$, where

$\lambda_{\mathrm{eff}} \equiv \lambda_{\mathrm{eff}}^{d}=\frac{c}{\omega_{p e}^{d}}=c \sqrt{\frac{m_{e} G_{0 d}}{4 \pi n_{0 d} e^{2}}}$.
Notice, that "effective" degenerate electron skin depth is related to the "effective" hot electron skin depth $\lambda_{\text {eff }}^{h}$ as

$\lambda_{\mathrm{eff}}^{d}=c \sqrt{\frac{\alpha G_{0 d}}{G_{0 h}}} \sqrt{\frac{m_{e} G_{0 h}}{4 \pi n_{0 h} e^{2}}}=\sqrt{\frac{\alpha G_{0 d}}{G_{0 h}}} \lambda_{\mathrm{eff}}^{h}$,

where

$\lambda_{\mathrm{eff}}^{h}=c \sqrt{\frac{m_{e} G_{0 h}}{4 \pi n_{0 h} e^{2}}}$.

Depending on $\alpha$, and the degeneracy level as well as the relativistic temperature of the fraction (outflow/jet), there are 2 drastically different length scales in addition to the conventional ion-skin depth,

$\lambda_{i}=\frac{c}{\omega_{p i}}=c \sqrt{\frac{m_{i}}{4 \pi n_{0 d} e^{2}}}$.

Here

$G_{0 d}\left(n_{0 d}\right)=\left[1+\left(R_{0 d}\right)^{2}\right]^{1 / 2}$

with

$$
R_{0 d}=\left(\frac{n_{0 d}}{n_{c}}\right)^{1 / 3}
$$

while (Mignone et al 2005; Ryu et al 2006)

$G_{0 h}=\frac{5}{2} \frac{T_{e 0}}{m_{e} c^{2}}+\frac{3}{2} \sqrt{\left(\frac{T_{e 0}}{m_{e} c^{2}}\right)^{2}+\frac{4}{9}}$.

One must emphasize that the intrinsic skin depths (the natural length scales of the dynamics), though over a large length span, are much shorter compared to the system size. For $d$ electrons, the effective mass goes from $G_{0 d}\left(n_{0 d}\right)=1+\frac{1}{2}\left(\frac{n_{0 d}}{n_{c}}\right)^{2 / 3}$ in the non-relativistic limit $\left(R_{0 d} \ll 1\right)$ to $G_{0 d}\left(n_{0 d}\right)=\left(\frac{n_{0 d}}{n_{c}}\right)^{1 / 3}$ in the ultrarelativistic regime $\left(R_{0 d} \gg 1\right)$, and for the $h$ component, the effective mass goes from $G_{0 h}\left(T_{e 0}\right)=1+\frac{5}{2} \frac{T_{e 0}}{m_{e} c^{2}}$ in the non-relativistic limit $\left(T_{e 0} \ll m_{e} c^{2}\right)$ to $G_{0 h}\left(T_{e 0}\right)=$ $4 \frac{T_{e 0}}{m_{e} c^{2}}$ in the ultra-relativistic regime $\left(T_{e 0} \gg m_{e} c^{2}\right)$.

By following the methodology of (Pino et al 2010) and (Berezhiani et al 2015a; Shatashvili et al 2016), we obtain the BB equilibrium conditions for both the $d$ and $h$ electrons (the primary difference is in the physics of $G_{d}$ and $G_{h}$ ). The Beltrami conditions

$$
\begin{aligned}
& \mathbf{B}-\nabla \times\left(G_{d} \gamma_{d} \mathbf{V}_{d}\right)=a_{d} \frac{n_{d}}{G_{d}}\left(G_{d} \gamma_{d} \mathbf{V}_{d}\right), \\
& \mathbf{B}-\nabla \times\left(G_{h} \gamma_{h} \mathbf{V}_{h}\right)=\alpha a_{h} \frac{n_{h}}{G_{h}}\left(G_{h} \gamma_{h} \mathbf{V}_{h}\right),
\end{aligned}
$$


align the Generalized vorticities along their velocity fields. This Beltrami alignment imposes (on the electron fluids) the following generalized Bernoulli Conditions, expressing the balance of all remaining potential forces,

$$
\nabla\left(G_{d} \gamma_{d}-\varphi\right)=0
$$

and

$$
\nabla\left(G_{h} \gamma_{h}-\varphi\right)=0
$$

may be combined to form

$$
G_{d} \gamma_{d}+G_{h} \gamma_{h}-2 \varphi=\text { const },
$$

where $\varphi$ is the electrostatic potential (of purely electromagnetic nature). This set, coupled with Ion fluid Beltrami Condition:

$\mathbf{B}+\zeta \nabla \times \mathbf{V}_{i}=(1+\alpha) a_{i} n_{i} \mathbf{V}_{i}, \quad \zeta=\left[G_{0}^{d} \frac{m_{e}^{d}}{m_{i}}\right]^{-1}$,

together with Ampere's law Eq.([6), defines the BB equilibrium states pertinent to the system of a two electron component $(d$ and $h$ ) fluid immersed in a neutralizing ion fluid. The separation (proportionality) constants $a_{d(h), i}$ are related to the system invariants, the total energy, and the generalized helicities for each component,

$h_{d(h), i}=\int\left(\operatorname{curl}^{-1} \boldsymbol{\Omega}_{d(h), i}\right) \cdot \boldsymbol{\Omega}_{d(h), i} d \mathbf{r}$.

Below we put $\varphi \equiv 0$ due to the quasi-neutrality hold throughout the overall dynamics assuming the incompressibility; gravity will be ignored for the time being.

The asymmetry between the bulk electron $(d)$ and the ion fluid is due to a small fraction of hot electrons $\left(\alpha \ll 1,\left|\mathbf{V}_{i}\right| \ll\left|\mathbf{V}_{d(h)}\right|\right)$. Notice that there are, in fact, two symmetry-breaking mechanisms in this model: 1) the first is due to different effective inertias for the $d$ and $h$ electrons, and 2) the second is from the small $\mathrm{h}$ contamination added to the bulk $d$ electrons $\left(\alpha \neq 0, \mathbf{V}_{h} \neq\right.$ $0)$. Each one of these is responsible for creating a net "current". The structure formation mechanism explored in (Mahaian et al 2009; Berezhiani et al 2010), (Steinhauer \& Ishida 1997), (Mahaian et al 2001), (Mahajan et al 2002), (Ohsaki et al 2001,2002)), originates, for instance, in the effective inertia difference. Asymmetry between the plasma constituents increases the number of conserved helicities, and eventually translates into a higher index Beltrami state (Mahajan \& Lingam 2015; Shatashvili et al 2016). It should also be mentioned that due to the different origin of relativistic effective masses $G_{d} \neq G_{h}$, the index of the Beltrami system is determined by the simultaneous action of both asymmetries.

\section{Quadrupole Beltrami Equations}

In this section we show that an appropriate but tedious manipulation of the set of the Eq.-s (6)-(18), leads us to an explicit quadruple Beltrami equation. The variable of choice turns out to be the Hot electron Fluid Velocity $\mathbf{V}_{h}$ (the Beltrami index is measured by the highest number of curl operators (Mahajan \& Lingam 2015)).

From Ampere's law in dimensionless form variables as:

$\left.\nabla \times \mathbf{B}=\left[(1+\alpha) \frac{N_{i}}{N_{0 i}} \mathbf{V}_{i}-\frac{N_{d}^{e}}{N_{0 d}^{e}} \mathbf{V}_{d}-\alpha \frac{N_{h}^{e}}{N_{0 h}^{e}} \mathbf{V}_{h}\right)\right]$,

we find that if $\alpha=0$ (no $h$ contamination, then quasineutrality reads $\left.N_{i}=N^{d}=N\right)$, we have:

$$
\mathbf{V}_{d} \equiv \mathbf{V}_{e}=\mathbf{V}_{i}-\frac{1}{N} \nabla \times \mathbf{B}
$$

that will reproduce the Double Beltrami (DB) state, relevant to an ion-degenerate electrons plasma

(Berezhiani et al 2015a). For $\alpha \neq 0$ and $\mathbf{V}_{i}=0$ (immobile ions), the relation

$$
\mathbf{V}_{d}=-\frac{N_{h}}{N_{d}} \mathbf{V}_{h}-\frac{1}{N_{d}^{e}} \nabla \times \mathbf{B}
$$

will lead to higher (Triple) Beltrami states when inertia effects in the degenerate and hot electron fluids are taken into account.

Observations show that hot electron fluid fraction can be small $(\alpha \ll 1)$; ion fluid velocity are also much smaller than those for lighter electron $(d, h)$ fluids $\left[\mathbf{V}_{i} \ll \mathbf{V}_{d}, \mathbf{V}_{h}\right]$. Thus, ion dynamics could be neglected in most of cases (Oliveira \& Tajima 1995) except for $\alpha=0$ when flow effects can be crucial in creating the structural richness in astrophysical environments, in the heating/cooling processes, and in Generalized Dynamo theory and flow acceleration phenomena (Mahaian et al 2001; Mahaian et al 2002; Mahajan et al 2005:2006), (Lingam \& Mahajan 2015).

For $\alpha=0$ (pure e-i plasma with degenerate electrons), it was shown that when electron inertia is neglected, the system reduces to a Double Beltrami state (Berezhiani et al 2015a). One could expect, then, that for the full model described in this paper, the composite Beltrami condition of index 4 will arise as two distinct "components" are being added. The index would fall to three when the ion flow effects are neglected $\left(\mathbf{V}_{i} \rightarrow 0\right)$. 
Let us now explore the new structures accessible to the Beltrami states for the full model - the $d$ and $h$ electrons and mobile ions. We will assume $\varphi \equiv 0$, make the simplifying assumption $\gamma_{d} \equiv 1, \gamma_{h} \equiv 1$ that reduces the Bernoulli Conditions (1516) to $G_{d}=$ const; $G_{h}=$ const . The resulting Ampere's law (20), in dimensionless variables, becomes

$$
\nabla \times \mathbf{B}=\left[(1+\alpha) \mathbf{V}_{i}-\mathbf{V}_{d}-\alpha \mathbf{V}_{h}\right]
$$

In terms of the bulk "Flow velocity" (combining $d$ electrons and ions),

$$
\mathbf{V}=\frac{1}{2}\left[(1+\alpha) \mathbf{V}_{i}+\mathbf{V}_{d}\right]
$$

one can express the Generalized ion Velocity and Momentum for $d$ electrons as $\left[\mathbf{P}_{d}^{e}=G_{0 d}^{e}\left(n_{0 d}^{e}\right) \mathbf{V}_{d}^{e}\right]$ :

$\mathbf{V}_{i}=\frac{1}{1+\alpha}\left(\mathbf{V}+\frac{1}{2} \nabla \times \mathbf{B}+\frac{\alpha}{2} \mathbf{V}_{h}\right)$

$\mathbf{P}_{d}=G_{0 d}^{e}\left(\mathbf{V}-\frac{1}{2} \nabla \times \mathbf{B}-\frac{\alpha}{2} \mathbf{V}_{h}\right)$

and the Ion flow Beltrami condition (18) as

$$
\mathbf{B}+\zeta \nabla \times \mathbf{V}_{i}=(1+\alpha) a_{i} \mathbf{V}_{i}
$$

Straightforward algebra, using Eqs. (23) and (24) in Eqs. (13) and (25), leads to $\left(G_{0 d}\left(n_{0 d}\right) \equiv G_{0}\right)$ :

$$
\begin{aligned}
\mathbf{V}= & \eta\left(\beta \nabla \times \nabla \times \mathbf{B}-\frac{1}{2}\left[a_{i}(1+\alpha)^{2} \beta-a_{d}\right] \nabla \times \mathbf{B}\right) \\
& +\eta[1+\beta(1+\alpha)] \mathbf{B}+\alpha \beta \nabla \times \mathbf{V}_{h}- \\
& -\frac{\alpha}{2}\left[a_{i}(1+\alpha)^{2} \beta-a_{d}\right] \mathbf{V}_{h}
\end{aligned}
$$

with $\eta \equiv\left[a_{i}(1+\alpha)^{2} \beta+a_{d}\right]^{-1} \quad, \quad \beta \equiv G_{0} \zeta^{-1}$.

The parameter $\beta$ is a measure of degeneracy as well as the mobility of ions: $\beta \rightarrow 0$ for immobile ion fluid $\left(m_{i} \rightarrow \infty\right)$ and $\beta \ll 1, R_{0 d} \ll 1 \quad\left[\beta>1, R_{0 d} \gg 1\right]$ for the weakly [strongly] degenerate electrons; in the latter case, the degenerate electron fluid inertia can not be ignored. In the limit of a pure e-i plasma $(\alpha \equiv$ $\left.0 ; \quad \beta \ll 1 ; \quad \eta \simeq a_{d}^{-1}\right)$, the pertinent simple relation $\mathbf{V}-\frac{1}{2} \nabla \times \mathbf{B}=a_{d}^{-1} \mathbf{B}$ reveals that the inertialess electrons move parallel to magnetic field.

Further manipulation of the system is displayed in Appendix A, the end result is the emergence of the quadruple Beltrami equation (QB) for $\mathbf{V}_{h}$ for arbitrary $\alpha\left[G_{h} \equiv G_{0 h}=H_{0}\right]$ :

$\eta G_{0} \nabla \times \nabla \times \nabla \times \nabla \times \mathbf{V}_{h}+\eta G_{0} b_{1} \nabla \times \nabla \times \nabla \times \mathbf{V}_{h}$

$+\eta b_{2} \nabla \times \nabla \times \mathbf{V}_{h}-b_{3} \nabla \times \mathbf{V}_{h}-2 \alpha b_{4} \mathbf{V}_{h}=0$.

Naturally such a system will be endowed with four distinct length (constructed from the defining parameters). Different effective masses of the degenerate bulk population and of a hot electron contamination and their ratio are the new elements of physics introduced in this paper. Notice that if either $\alpha$ ( $h$ fraction) or the $b_{4}$ (ion mobility factor) were zero, the Beltrami index of the system goes down implying the disappearance of a scale length.

Solving the Eq.(27) for $\mathbf{V}_{h}$ and plugging it into (14) we will get the equation for $\mathbf{B}$; for the pure incompressible degenerate e-i plasma it is better to use Eq. A1 (with $\alpha \equiv 0$ ) directly to find the magnetic field $\mathbf{B}$.

\subsection{Assymetry Induced Macroscopic Structure Formation}

Justified by observational evidence (see introduction), we will assume $\alpha \ll 1$ that will simplify the coefficients in Eq. (27). A formal factorization of (27) leads to

$\left(\operatorname{curl}-\mu_{1}\right)\left(\operatorname{curl}-\mu_{2}\right)\left(\operatorname{curl}-\mu_{3}\right)\left(\operatorname{curl}-\mu_{4}\right) \mathbf{V}_{i}=0$,

where the inverse length scales $\mu_{i}$ are functions of $\alpha, \beta, n_{0 d}, H_{0}$ and the degeneracy-determined mass factor $G_{0}$. The general solution of Eq.(28) is a sum of four Beltrami fields $\mathbf{F}_{\mathbf{k}}$ (solutions of Beltrami Equations $\left.\nabla \times \mathbf{F}_{k}=\mu_{k} \mathbf{F}_{k}\right)$ while eigenvalues $\left(\mu_{k}\right)$ of the curl operator are the solutions of the fourth order equation

$$
\mu^{4}-b_{1}^{*} \mu^{3}+b_{2}^{*} \mu^{2}-b_{3}^{*} \mu+b_{4}^{*}=0 .
$$

Details of a similar analysis can be found in (Shatashvili et al 2016). The interesting and important result of this enquiry follow after an examination of the various $b^{*}$ coefficients of (29).

Though the inverse scales, determined by $b_{1}^{*}, b_{2}^{*}$, and $b_{3}^{*}$, do get slightly modified by $\alpha \ll 1$ corrections, it is the inverse scale associated with $b_{4}^{*}$ that is most profoundly affected; being proportional to $\alpha$, it tends to become small, i.e, the corresponding scale length becomes large as $\alpha$ approaches zero; the corresponding scale length becomes strictly infinite for $\alpha=0$, and disappears reducing (29) to a triple Beltrami system.

Thus the asymmetry induced due to the small fraction of relativistically hot electrons may lead to the formation of macroscopic structures through creating an intermediate/large length scale, much larger than 
the intrinsic scale skin depths (but less than the system size). It is important to note that this mechanism operates for all levels of bulk electron as long as degeneracy (the range of $G_{0}$ was irrelevant) and the hot electron fraction is nonzero. The possible significance and importance of natural mechanisms of this sort (such cases are natural in astrophysical conditions as discussed in the introduction) for creating Macro-structures in astrophysical objects was already discussed in (Shatashvili et al 2016) for different type asymmetric multi-fluid systems.

\section{Scale Hierarchy in 2-temperature relativistic e-i plasmas}

The new macroscopic scale discussed in previous section can be "determined" by dominant balance arguments: As the scale gets larger, $|\nabla|$ gets smaller, and the dominant balance will be between the last terms of (29), yielding [we remind the reader, that all lenghts are normalized to the $\lambda_{\text {eff }}$, and $\zeta \gg 1$ even for ultrarelativistic case]:

$$
L_{\text {macro }}=\frac{1}{2 \alpha} \frac{\left|b_{3}\right|}{\left|b_{4}\right|}=\frac{C}{2 \alpha}
$$

where $C\left(a_{i}, a_{d}, a_{h}, G_{0}, H_{0}, \beta\right)$ is a rather complicated function of the plasma-system parameters (see Appendix A for $b$-coefficients).

Let us assume that the densities of the 2-temperature relativistic electron-ion plasmas of interest are such that $(1-\beta) \gg \alpha(\beta \leq 1$ even for the ultra-relativistic case when the degenerate electron component density range is within $\left.\left(10^{25}-10^{34}\right) \mathrm{cm}^{-3}\right)$. We can, then simplify $C$ for $\left(a_{d} / a_{i}\right) \leq \beta,\left(a_{h} / a_{i}\right) \leq \beta$ and write

$$
L_{\text {macro }} \sim \frac{H_{0}}{\alpha\left[\beta+a_{h}(1-\beta)\right]}|(1-\beta)| .
$$

There are 2 possible, observationally relevant, limiting cases:

(i) The degenerate electron fluid density is so high ( $10^{33}-10^{34} \mathrm{~cm}^{-3}$, strongly relativistic Fermi energy) that $\beta$ approaches unity [typically $0.1-0.5$ ]. The macroscopic length (31), then, yields,

$$
L_{\text {macro }} \sim \frac{H_{0}}{\alpha} \frac{1-\beta}{\beta} \gg 1 .
$$

(ii) The degenerate electron fluid density is in the lower range $\left(\sim 10^{25}-10^{32} \mathrm{~cm}^{-3}\right)$ leading to a $\beta \ll 1$. The expression for $L_{\text {macro }}$ simplifies to

$$
L_{\text {macro }} \geq \frac{H_{0}}{\alpha} \frac{1}{a_{h}} \gg 1
$$

for any $H_{0}>1$ and $G_{0}>1$.

Notice that the hot electron induced $L_{\text {macro }}$ for the strongly degenerate bulk electrons tends to be smaller than the corresponding length for low bulk degeneracy.

It is important to consider another obviously interesting $\beta \ll 1$ (weakly degenerate) case when $a_{d} \sim a_{h} \equiv a$ and they are both $\sim a_{i}$ (note that under the same assumptions $\left.\eta \sim a^{-1}\right)$. The $b^{*}$-coefficients take the form (reminding that $\beta^{-1} G_{0}=\zeta \gg 1$ ):

$b_{1}^{*}=\zeta a, \quad b_{2}^{*}=2 H_{0} G_{0}^{-1}\left(1+0.5 a^{2}\right)+\alpha \zeta a^{2} ;$

$b_{3}^{*}=-\alpha a\left[\zeta+G_{0}^{-1}\left(1+0.5 a^{2}\right)\right] ; b_{4}^{*}=\frac{\alpha}{2} G_{0} a^{2}(1-a)$.

We see that depending on the physical parameters: $H_{0}, G_{0}, \alpha, \zeta$, different scale hierarchies will emerge.

Even at a very small fraction of the hot electron fluid $(\alpha \ll 1)$, none of $b^{*}$-coefficients vanish - they remain finite and the macro/meso scale is always present in such a system. Then, for $a \gg 1(a \ll 1)$, we will have quadruple (triple) Beltrami states in our complex relativistic system. When $a \equiv 1$ we have a TripleBeltrami equilibrium. If in addition, the $h$ electron fraction vanishes $(\alpha \equiv 0)$, the equilibrium reduces to a Double-Beltrami state consistent to previous results (Berezhiani et al 2015a).

The three component plasma (ions, and two species of electrons), studied in this paper, is another example of the rule that the associated $\mathrm{BB}$ equilibria follow, i.e, the Beltrami index is $I=M+1$, where $M$ is the number of "independent" components. Naturally the index is a measure of the independent characteristic scale lengths [see Mahajan \& Lingam 2015].

The scale hierarchy, pertinent to our model of astrophysical significance, may be summarized as follows:

(1) For an ion-degenerate electron plasma, the equilibrium is triple Beltrami with the following fundamental three scales; the system size $L$, and the two intrinsic scales, the $d$-electron and ion skin depths. If $d$ electron inertia is negligible (relatively lower densities), then equilibrium collapses to a double Beltrami.

(2) Both the skin depths associated with $d$ and $h$, that are microscopic in a non degenerate/nonrelativistic plasma, can become larger due to relativistic effects and could be classified as meso-scales $\left[l_{\text {meso }}\right]$. Under some special constraints on the Bertrami parameters, the meso-scale $l_{\text {meso }}$ can become very large!

(3) With the relativistically hot electron $(h)$ species acting as an independent component, the equilibrium becomes quadruple Beltrami with a new additional 
scale, $L_{\text {macro }}$ Originating entirely in the $h$ fraction $(\alpha \neq 0)$, this scale disappears as this fraction goes to zero. Both the larger ion mass and lower density hot electron fraction contribute towards boosting $L_{\text {macro }}$.

(4) In the limit of immobile ions (only $d$ and $h$ electrons are the dynamical components), the equilibrium is triple Beltrami (as expected), and the largest intrinsic scales are the relativistically enhanced skin-depths of the two species, the relative scale size will be determined by the ratio $G_{0} / H_{0}$.

\section{Summary}

We studied the quasi equilibrium Beltrami-Bernoulli states that are accessible to a three component plasma composed of two electron species (a highly degenerate main component mixed with a smaller classical relativistic hot flow) immersed in a neutralizing ion background; the latter could be either static or dynamic. Study of such a plasma could be generally relevant to the evolution of certain astrophysical objects, specifically during the accretion stage of stars; star collapsing etc. The two electron species - the bulk degenerate electron fluid $(d)$ and a small contamination of relativistically hot electrons $(h)$ - contribute two components to a three component plasma that in addition, has a neutralizing ion background.

The $h$ contamination has the expected but striking effect of providing an added macroscopic scale lying between the system size and the relatively small intrinsic scales (measured by the skin depths). The existence of a new scale for structure formation may provide crucial insights into the evolution of accreting stars; in particular, new channels for energy transformations may become available [(Mahajan et al 2002; Ohsaki et al 2001,2002; Mahajan et al 2005:2006), (Shiraishi et al 2009), (Shatashvili \& Yoshida 2011)].

Although not the direct subject matter of this paper, the existence of a small relativistic electron component is reminiscent of the runaway electron population in a Tokamak. It is conceivable that the energy exchange processes studied in this paper, could have some relevance to runaway induced phenomena.

The new element of physics introduced in this paper arises due to the different effective masses of the $d$ and $h$ electrons. In fact, that is what forces us to treat them as independent components. It is this piece of physics that leads to the creation of the $L_{\text {macro }}\left[l_{\text {meso }}\right]$ alluded to on the preceding paragraph.

These macro/intermediate scales, opening new pathways for energy transformations, can advance our understanding of a host of quiescent as well as explosive astrophysical phenomena - magnetic field generation, structure formation, fast/transient outflow and jet formation, heating/cooling etc. We plan to explore the consequences of the particular findings of this paper in the context of the accreting White Dwarf evolution problem, and the events accompanying the phenomenon of star-collapse.

Finally, when a complex system moves from Doubleto Triple- and Quadruple-Beltrami states, the roots (inverse length-scales) will exhibit a wide range of behavior. In the quadruple case, there are possible transitions from 2 complex-conjugate pairs to: 1) one complexconjugate pair and two real roots, and 2) to 4 real roots. In each of these cases, the conversion of magnetic energy into flow energy can occur; the process will result in energy transfer similar to what is commonly associated with magnetic reconnection. Such scenarios may explain explosive/eruptive phenomena like magnetar giant flares; like outflows in WD atmospheres.

\section{Acknowledgements}

Authors acknowledge the support from Shota Rustaveli Georgian National Foundation Grant Project No. FR17-391. Work of SMM was supported by US DOE Contract No.DE-FG02-04ER54742.

\section{References}

Asenjo, F.A. and Mahajan, S.M. Physica Scripta, 9(1), 015001 (2015).

Barnaveli, A. and Shatashvili, N.L. AAS, 362, 164, (2107).

Begelman, M.C., Blandford, R.D., and Rees, M.D. Rev. Mod. Phys. 56255 (1984).

Berezhiani, V.I. and Mahajan, S.M. Phys. Rev. Lett. 73, 1110 (1994); Phys. Rev. E 521968 (1995).

Berezhiani, V.I., Mahajan, S.M. and Shatashvili, N.L. Phys. Rev. A81, 053812 (2010); ibid. J. of Plasma Phys. 76, 467 (2010).

Berezhiani, V.I., Shatashvili, N.L. and Mahajan, S.M. Phys. Plasmas 22, 022902 (2015a).

Berezhiani, V.I., Shatashvili, N.L. and Tsintsadze, N.l. Physica Scripta 90(6), 068005 (2015b).

Bhattacharjee, C., Das, R., Stark, D., Mahajan, S.M. Phys. Rev. E, P92(6), 063104 (2015).

Cercignani, C. and Kremer, G.M. 2002 The relativistic Boltzmann equation: theory and applications Birkhäuser, Basel; chapter 3.

Dennis, G.R., Hudson, S.R., Dewar, R.L. and Hole, M.J. Phys. Plasmas 21, 042501 (2014).

Gomez, D.O., Kandus, Mon. Not. R. Astron. Soc.481(3), 3988 (2018).

Hollands, M., Gaensicke, B., \& Koester, D. MNRAS, 450, 68 (2015). 
Kawka, A., Vennes, S., Schmidt, G. D., Wickramasinghe, D. T., \& Koch, R. Astrophys. J., 654, 499 (2007).

Iqbal, N., Berezhiani, V.I. and Yoshida, Z. Phys. Plasmas 15, 032905 (2008).

Kawka, A. and Vennes, S. MNRAS 439, L90 (2014).

Kepler, S. O., Pelisoli, I., Jordan, S., Kleinman, S.J., Koester, D., Külebi, D.B., Pecanha, B.V., Castanheira, B.G., Nitta, A., Costa, J.E.S., Winget, D.E., Kanaan, A. and Fraga, L. MNRAS 429, 2934 (2013).

Kryvdyk, V. Mon. Not. R. Astron. Soc., 309, 593 (1999).

Kryvdyk, V and Agapitov, A. 15th European Workshop on White Dwarfs ASP Conference Series, 372, 411 (2007).

Koester, D. and Chanmugam,G. Rep. Prog. Phys. 53, 837 (1990).

Külebi, B., Jordan, S., Euchner, F., Gnsicke, B. T., \& Hirsch, H. A\&A, 506, 1341 (2009).

Liebert, J., Bergeron, P., \& Holberg, J. B. AJ, 125, 348 (2003).

Lingam, M. and Mahajan, S.M. Mon. Not. R. Astron. Soc.449, L36 (2015).

Mahajan, S.M. and Yoshida, Z. Phys. Rev. Lett.81, 4863 (1998).

Mahajan, S.M., Miklaszewski, R., Nikolskaya, K.I. and Shatashvili, N.L. Phys. Plasmas 8, 1340 (2001).

Mahajan, S.M., Nikolskaya, K. I., Shatashvili, N.L. and Yoshida, Z. Astrophys. J.576, L161 (2002).

Mahajan, S.M. Phys. Rev. Lett.90, 035001 (2003).

Mahajan, S.M. Phys. Plasmas, 23(11), 112104 (2016).

Mahajan, S.M. and Asenjo, F.A. Phys. Rev. Lett., 107(19) id. 195003 (2011).

Mahajan, S.M., Shatashvili, N.L., Mikeladze, S.V. and Sigua, K.I. Astrophys. J.634, 419 (2005); Phys. Plasmas 13, 062902 (2006).

Mahajan, S.M., Shatashvili, N.L. and Berezhiani, V.I. Phys. Rev. E80, 066404 (2009).

Mahajan, S.M. and Lingam, M. Phys. Plasmas, 22(9), 092123 (2015).

Michel, F.C. Theory of Neutron Star Magnetospheres, University of Chicago Press, Chicago, (1991).

Michel, F.C. Rev. Mod. Phys. 54, 1 (1982).

Mignone, A., Plewa, T. \& Bodo, G. Astrophy. J. Supp. 160, 199 (2005).

Morrison, P.J. Rev. Mod. Phys. 70, 467 (1998).

Mukai, K. PASP 129, 062001 (2017)

Oliveira, S.R. and Tajima, T. Phys. Rev. E52, 287 (1995).

Ohsaki, S., Shatashvili, N.L., Yoshida, Z. and Mahajan, S.M. Astrophys. J.559, L61 (2001); S. Ohsaki, N.L. Shatashvili, Z. Yoshida and S.M. Mahajan, Astrophys. J.570, 395 (2002).

Pino, J., Li, H. and Mahajan, S.M. Phys. Plasmas 17, 112112 (2010).

Ryu, D., Chattopadhyay, I \& Choi, E. J. Korean Phys. Soc. 49(4), 1842 (2006).

Schmidt, G. D., Harris, H. C., Liebert, J., et al. Astrophys. J., 595, 1101 (2003).

Shapiro, L. and Teukolsky, S.A. Black Holes, White Dwarfs and Neutron Stars: The Physics of Compact Objects, (John Wiley and Sons, New York, 1973).

Shatashvili, N.L., Mahajan, S.M. and Berezhiani, V.I. AAS, 361, 70 (2016).
Shatashvili, N.L. and Yoshida, Z. AIP Conf. Proc. 1392, 73 (2011).

Shiraishi, J, Yoshida, Z. and Furukawa, M. Astrophys. J., 697, 100 (2009).

Steinhauer, L.C. and Ishida, A. Phys. Rev. Lett.79, 3423 (1997).

Tremblay, P.-E., Fontaine, G., Freytag, B., Steiner, O., Ludwig, H.-G., Steffen, M., Wedemeyer, S. and Brassard, P. Astrophys. J., 812, 19, (2015).

Winget, D. E. and Kepler, S. O. Annu. Rev. A\&A 46, 157 (2008).

Yoshida, Z. and Mahajan, S.M. J. Math. Phys. 40, 5080 (1999).

Yoshida, Z., Mahajan, S.M., Ohsaki, S., Iqbal, M. \& Shatashvili, N.L. Phys. Plasmas, 8(5), 2125 (2001).

This 2-column preprint was prepared with the AAS LATEX macros v5.2. 
A Appendix - Deriving Quadruple Beltrami Equation and Its Analysis

Plugging Eq. (26) into the Eq.-s (23124) and then using them in Eq. (14), and after some tedious algebra, we get

$$
\begin{aligned}
2 \eta G_{0} \nabla \times \nabla \times \nabla \times \mathbf{B} & +\eta G_{0} \alpha_{1} \nabla \times \nabla \times \mathbf{B}+2 \eta \alpha_{2} \nabla \times \mathbf{B}-2 \alpha_{3} \mathbf{B}= \\
& =-2 \alpha G_{0} \nabla \times \nabla \times \mathbf{V}_{h}-\alpha \beta^{-1} G_{0}^{2} \alpha_{4} \nabla \times \mathbf{V}_{h}+\frac{\alpha}{2} \beta^{-1} \alpha_{5} \mathbf{V}_{h},
\end{aligned}
$$

where

$$
\begin{aligned}
& \alpha_{1}=a_{d}\left[1+\beta^{-1} G_{0}\right]+a_{i}(1+\alpha) \beta\left[1+(1+\alpha) \beta^{-1} G_{0}\right], \quad \alpha_{2}=[1+(1+\alpha) \beta]+\frac{1}{2}(1+\alpha) a_{d} a_{i}, \\
& \alpha_{3}=\left(a_{i}-a_{d}\right)+\alpha(1-\alpha-\beta) a_{i}, \quad \alpha_{4}=\left(1-\beta G_{0}^{-1}\left[a_{d}+a_{i}(1+\alpha)\right]\right), \\
& \alpha_{5}=\left[a_{d}+a_{i}(1+\alpha) \beta\right]+\eta^{-1}\left[a_{d}-a_{i}(1+\alpha) \beta\right]-2 a_{d}\left[a_{d}-a_{i}(1+\alpha) \beta\right] .
\end{aligned}
$$

The equation (A1) with (A2) for no hot electron-fluid fraction $(\alpha \equiv 0)$ will eventually give the so called "Triple Beltrami" equation for the magnetic field B (i.e. l.h.s. of Eq. A1 $\equiv 0)$ for e-i plasma with degenerate electrons. While for classical pure e-i plasma we see that $\alpha_{1} \rightarrow \zeta a_{d}$ and the 2nd term in l.h.s. of Eq. A1 can become much bigger than the 1st term $(\zeta \gg 1)$ and one obtains the so called double-curl equation leading to Double Beltrami States.

Using Eq. (14) in eq. (A1), one obtains for arbitrary $\alpha\left[H_{h} \equiv H_{0 h}=H_{0}\right.$, we do not study the heating/cooling problem] the quadruple Beltrami equation $(\mathrm{QB})$ for $\mathbf{V}_{h}$ :

$\eta G_{0} \nabla \times \nabla \times \nabla \times \nabla \times \mathbf{V}_{h}+\eta G_{0} b_{1} \nabla \times \nabla \times \nabla \times \mathbf{V}_{h}+\eta b_{2} \nabla \times \nabla \times \mathbf{V}_{h}-b_{3} \nabla \times \mathbf{V}_{h}-2 \alpha b_{4} \mathbf{V}_{h}=0$,

where

$$
\begin{aligned}
& b_{1}=\alpha_{1}+2 \alpha a_{h} ; \quad b_{2}=2 \alpha H_{0}+2 \alpha G_{0}\left(\alpha_{1} a_{h}+2 \eta^{-1}\right) ; \\
& b_{3}=2 H_{0} \alpha_{3}-\alpha\left(\beta^{-1} G_{0}^{2} \alpha_{4}+2 \eta \alpha_{2} a_{h}\right) ; \quad b_{4}=\frac{1}{4} \beta^{-1} \alpha_{5}+\alpha_{3} a_{h} .
\end{aligned}
$$

It is clear that the large scale is automatically introduced into the system since $b_{4} \neq 0, \alpha \neq 0$ in Eq. A3). Solving the Eq. (A3) for $\mathbf{V}_{h}$ and plugging it into (14) we will get the equation for $\mathbf{B}$.

Assuming $\alpha \ll 1$ for our problem of study one can simplify the coefficients in Eq. A3 as follows:

$$
\begin{aligned}
& \eta=\left(a_{d}+\beta a_{i}\right)^{-1} ; \quad \alpha_{1}=\left(1+\beta^{-1} G_{0}\right)\left(a_{d}+\beta a_{i}\right) ; \quad \alpha_{2}=\left[(1+\beta)+\frac{1}{2} a_{d} a_{i}\right] \\
& \alpha_{3}=a_{i}-a_{d} ; \quad \alpha_{4}=\left[1-\beta G_{0}^{-1}\left(a_{i}+a_{d}\right)\right] ; \quad \alpha_{5}=\left[\left(a_{d}+\beta a_{i}\right)-\left(a_{d}-\beta a_{i}\right)^{2}\right]
\end{aligned}
$$

leading to:

$$
\begin{aligned}
& b_{1}=\left(1+\beta^{-1} G_{0}\right) a_{d}+(1+\beta) G_{0} a_{i}+2 \alpha a_{h} \\
& b_{2}=2 H_{0}\left[(1+\beta)+0.5 a_{d} a_{i}\right]+\alpha G_{0}\left[\left(1+\beta^{-1} G_{0}\right) a_{d} a_{h}+\left(G_{0}+3 \beta\right)+2 a_{d}\right] \\
& b_{3}=2 H_{0}\left(a_{i}-a_{d}\right)-\alpha\left[\beta^{-1} G_{0}^{2}-G_{0}\left(a_{i}+a_{d}\right)\right]-\alpha \frac{2 a_{h}}{a_{d}+\beta a_{i}}\left[(1+\beta)+0.5 a_{d} a_{i}\right]
\end{aligned}
$$




$$
b_{4}=\frac{1}{4}\left[a_{d}\left(1-a_{d}\right)+\beta a_{i}\left(1-\beta a_{i}\right)+2 \beta a_{i} a_{d}\right]+\left(a_{i}-a_{d}\right) a_{h} .
$$

The quadruple Beltrami Equation (A3) can be factorized as

$$
\left(\operatorname{curl}-\mu_{1}\right)\left(\operatorname{curl}-\mu_{2}\right)\left(\operatorname{curl}-\mu_{3}\right)\left(\operatorname{curl}-\mu_{4}\right) \mathbf{V}_{i}=0
$$

where $\mu_{i}$-s define the coefficients in Eq.(27) and are the functions of $\alpha, \beta, n_{0 d}, H_{0}$ and the degeneracydetermined mass factor $G_{0}$. The general solution of Eq.(28) is a sum of four Beltrami fields $\mathbf{F}_{\mathbf{k}}$ (solutions of Beltrami Equations $\left.\nabla \times \mathbf{F}_{k}=\mu_{k} \mathbf{F}\right)$ while eigenvalues $\left(\mu_{k}\right)$ of the curl operator are the solutions of the fourth order equation

$$
\mu^{4}-b_{1}^{*} \mu^{3}+b_{2}^{*} \mu^{2}-b_{3}^{*} \mu+b_{4}^{*}=0,
$$

where

$$
b_{1}^{*}=b_{1} ; \quad b_{2}^{*}=G_{0}^{-1} b_{2} ; \quad b_{3}^{*}=\left(\eta G_{0}\right)^{-1} b_{3} ; \quad b_{4}^{*}=2 \alpha\left(\eta G_{0}\right)^{-1} b_{4}
$$

The details of analysis of above equation can be found in (Shatashvili et al 2016) for different physical system. Such analysis shows that for a rather big range of parameters there is a guaranteed scale separation in 2-temperature relativistic e-i plasma with degenerate (bulk) electrons and small fraction of relativistically hot electrons. 